\title{
THE $p$-PERIODICITY OF THE MAPPING CLASS GROUP AND THE ESTIMATE OF ITS $p$-PERIOD
}

\author{
YINING XIA \\ (Communicated by Frederick R. Cohen)
}

\begin{abstract}
We determine completely the primes $p$ for which the Farrell-Tate cohomology of the mapping class group $\Gamma_{g}$ is $p$-periodic. We also estimate the $p$-period of a $p$-periodic $\Gamma_{g}$.
\end{abstract}

For $\Gamma$ any group of finite virtual cohomological dimension (vcd) and a prime $p$, we say that the group $\Gamma$ has $p$-periodic cohomology if there exists a positive integer $d$ such that the Farrell-Tate cohomology groups $\widehat{H}^{i}(\Gamma ; M)$ and $\widehat{H}^{i+d}(\Gamma ; M)$ have naturally isomorphic $p$-primary components for all $i \in Z$ and $Z \Gamma$-modules $M$. The $p$-period of $\Gamma$ is defined as the least value of $d$ [B1].

Recall that the mapping class group $\Gamma_{g}$ is defined to be the group of path components of orientation-preserving homeomorphisms of the orientable closed surface $S_{g}$ of genus $g$. We always assume $g>1$. It is well known that the mapping class group $\Gamma_{g}$ is of finite vcd and the $\operatorname{vcd}\left(\Gamma_{g}\right)=4 g-5[\mathrm{H}]$.

In this paper, we determine completely the primes $p$ for which $\Gamma_{g}$ is $p$ periodic. Furthermore, we estimate the $p$-period of a $p$-periodic $\Gamma_{g}$ by using the $p$-period of a metacyclic subgroup of $\Gamma_{g}$ as a lower bound and a homogeneous Chern class polynomial of the canonical homology representation $\Gamma_{g} \rightarrow \mathrm{GL}(2 g, Z)$ as an upper bound. The main results are as follows:

Theorem 1. (a) The mapping class group $\Gamma_{g}$ is never 2-periodic.

(b) The mapping class group $\Gamma_{k p+i}$ is always p-periodic when $i \neq 1 \bmod (p)$ where $p$ is an odd prime and $k \geq 0$.

(c) The mapping class group $\Gamma_{k p+1}$ is p-periodic if and only if the interval $[(2 k+3) / p,(2 k+2) /(p-1)]$ does not contain an integer and $k \neq 0,-1$ $\bmod (p)$ where $p$ is an odd prime. In particular, $\Gamma_{k p+1}$ can be p-periodic only when $k \leq\left(p^{2}-5\right) / 2$.

Theorem 2. If $k \neq 0 \bmod (p)$ and $p>2$, then $\Gamma_{(p-1)(k p-k-2) / 2}$ is $p$-periodic and the p-period of $\Gamma_{(p-1)(k p-k-2) / 2}$ is a multiple of $2(p-1)$. Moreover, if $k<(p-1) / 2$, the $p$-period of $\Gamma_{(p-1)(k p-k-2) / 2}$ equals $2(p-1)$.

Theorem 3. If $3 \leq d$ and $d$ divides $p-1$, then $\Gamma_{(p-1)(d-2) / 2}$ is p-periodic and the p-period of $\Gamma_{(p-1)(d-2) / 2}$ is a multiple of $2 d$.

Received by the editors February 28, 1991.

1991 Mathematics Subject Classification. Primary 57R20, 20F38, $20 \mathrm{~J} 10$.

(C) 1992 American Mathematical Society $0002-9939 / 92 \$ 1.00+\$ .25$ per page 
Theorem 4. Let $2 g-2=m p-i$ where $0 \leq i \leq p-1, p$ is an odd prime, and $p^{r-1} \leq m \leq p^{r}$. Assume that $\Gamma_{g}$ is p-periodic. Then

(a) If $[2 g /(p-1)]<p^{r}$, then the $p$-period of $\Gamma_{g}$ divides $2 p^{r-1}(p-1)$.

(b) If $[2 g /(p-1)] \geq p^{r}$, then the p-period of $\Gamma_{g}$ divides $2 p^{r}(p-1)^{2}$.

The remainder of this paper is organized as follows: In the first section we prove Theorem 1. In $\S 2$ we give a lower bound for the $p$-period of $\Gamma_{g}$ for $g=k(p-1)$ and thus prove Theorem 3. In $\S 3$ we give an upper bound for the $p$-period of $\Gamma_{g}$ for $g=k(p-1)$ and thus prove Theorem 2. In $\S 4$ we give an upper bound for the $p$-period of $\Gamma_{g}$ when $\Gamma_{g}$ is any $p$-periodic mapping class group and thus prove Theorem 4.

\section{The $p$-PERIODICITY OF THE MAPPING ClasS GROUP $\Gamma_{g}$}

For a group $\Gamma$ of finite vcd, recall that $\Gamma$ is $p$-periodic if and only if $\Gamma$ does not contain $Z / p \times Z / p$ [B1]. Furthermore, the positive solution of the Nielsen conjecture by Kerckhoff $[\mathrm{K}]$ implies that the finite group $F$ is a subgroup of $\Gamma_{g}$ if and only if $F$ is isomorphic to a subgroup of $\mathrm{Homeo}^{+}\left(S_{g}\right)$, the group of orientation preserving homeomorphisms of $S_{g}$.

Proposition 1.1. The finite group $F$ is isomorphic to a subgroup of $\mathrm{Homeo}^{+}\left(S_{g}\right)$ with branching data $\left(h ; n_{1} \cdots n_{b}\right)$ if and only if $F$ satisfies the following conditions:

(1) $F=\left\langle a_{1}, \ldots, a_{h} ; b_{1}, \ldots, b_{h} ; c_{1}, \ldots, c_{b}\right\rangle$;

(2) $\prod_{1 \leq i \leq h}\left[a_{i}, b_{i}\right] \prod_{i \leq j \leq b} c_{j}=1$;

(3) $\operatorname{Order}\left(c_{i}\right)=n_{i}$;

(4) Riemann-Hurwitz equation $2 g-2=|F|(2 h-2)+|F| \sum_{1 \leq i \leq b}\left(1-1 / n_{i}\right)$.

Proof. See [T].

Lemma 1.2. Let $G$ be a finite subgroup of $\operatorname{Homeo}^{+}\left(S_{g}\right)$. Then $G$ is also isomorphic to a subgroup of $\mathrm{Homeo}^{+}\left(S_{g+k|G|}\right)$. Here $k$ is a nonnegative integer.

Proof. It follows immediately by Proposition 1.1.

Proof of Theorem 1. (a) We only need to show $\Gamma_{i} \supset Z / 2 \times Z / 2$ for $2 \leq i \leq 5$ by Lemma 1.2. In fact, write $Z / 2 \times Z / 2=\left\langle x, y \mid x^{2}=y^{2}=1, x y=y x\right\rangle$ in the following forms:

(i) when $g=2, Z / 2 \times Z / 2=\langle x, x, x, y, x y\rangle, h=0, b=5$;

(ii) when $g=3, Z / 2 \times Z / 2=\langle x ; y ; x, x\rangle, h=1, b=2$;

(iii) when $g=4, Z / 2 \times Z / 2=\langle x ; y ; x, y, x y\rangle, h=1, b=3$;

(iv) when $g=5, Z / 2 \times Z / 2=\langle x ; y ; x, y, x, y\rangle, h=1, b=4$.

It is straightforward to check by Proposition 1.1 that (i) $\Gamma_{2} \supset Z / 2 \times Z / 2$ with branching data $(0 ; 2,2,2,2,2)$, (ii) $\Gamma_{3} \supset Z / 2 \times Z / 2$ with branching data $(1 ; 2,2)$, (iii) $\Gamma_{4} \supset Z / 2 \times Z / 2$ with branching data $(1 ; 2,2,2)$, and (iv) $\Gamma_{5} \supset Z / 2 \times Z / 2$ with branching data $(1 ; 2,2,2,2)$. We have proved that $\Gamma_{2}$ is never 2-periodic.

In the rest of this paper, $p$ is an odd prime.

(b) $\Gamma_{k p+i}$ is $p$-periodic for $i \neq 1$. Otherwise, we have $\Gamma_{k p+i} \supset Z / p \times Z / p$. The Riemann-Hurwitz formula holds: $2(k p+i)-2=p^{2}(2 h-2)+p^{2}(1-1 / p) b$, i.e., $2 k+(2 i-2) / p=p(2 h-2)+(p-1) b$ implies $2 i-2=0 \bmod (p)$, forcing $i=1 \bmod (p)$. This is a contradiction. 
(c) Claim 1. If $k=0,-1 \bmod (p)$ or the interval $[(2 k+3) / p$, $(2 k+2) /(p-1)]$ contains an integer, then $\Gamma_{k p+1} \supset Z / p \otimes Z / p$.

Case $1 . k=0 \bmod (p)$. Suppose $k=n p$, where $n$ is a nonnegative integer. We show a $Z / p \times Z / p$ free action on $S_{k p+1}$ by Proposition 1.1. In fact, write $Z / p \times Z / p=\left\langle x, y \mid x^{p}=y^{p}=1 x y=y x\right\rangle=\left\langle x_{1}, x_{2}, \ldots, x_{n+1}\right.$; $\left.y_{1}, y_{2}, \ldots, y_{n+1}\right\rangle, h=0, b=n+1$, where $x_{i}=x$ and $y_{i}=y, 1 \leq i \leq n+1$. Notice that the Riemann-Hurwitz formula $2(k p+1)-2=p^{2}(2(n+1)-2)$ holds.

Case 2. $k=-1 \bmod (p)$. We show that there is a $Z / p \times Z / p$ action with two singular points on $S_{k p+1}$ by Proposition 1.1. Write

$$
Z / p \times Z / p=\left\langle x_{1}, x_{2}, \ldots, x_{n} ; y_{1}, y_{2}, \ldots, y_{n} ; x, x^{-1}\right\rangle,
$$

$h=n, b=2$, where $n=(k+1) / p \geq 1, x_{i}=x, y_{i}=y, 1 \leq i \leq n$. Notice that the Riemann-Hurwitz formula $2(k p+1)-2=p^{2} 2(n-1)+p^{2} 2(1-1 / p)$ holds.

Case 3. The interval $[(2 k+3) / p,(2 k+2) /(p-1)]$ contains an integer $n$. We show that there is a $Z / p \times Z / p$ action on $S_{k p+1}$ with $t=n p-2 k$ singular points. Note $t=n p-2 k \geq 3$.

Let $h=k+1-n(p-1) / 2$, then $h \geq k+1-(2 k+2) / 2=0$. Write $Z / p \times Z / p=$ $\left\langle x_{1}, x_{2}, \ldots, x_{h} ; y_{1}, y_{2}, \ldots, y_{h} ; \bar{y}, x_{1}, x_{2}, \ldots, x_{t-2},\left(\prod_{1 \leq j \leq t-2} x_{j}\right)^{-1} y^{-1}\right\rangle$. Here $x_{i}=x_{j}=x$ and $y_{i}=y, 1 \leq i \leq h, 1 \leq j \leq t-2$. Note that the Riemann-Hurwitz formula $2(k p+1)-2=p^{2}(2 h-2)+p^{2}(1-1 / p) t$ holds, i.e., $2 k p=p^{2}(2 k-n(p-1))+p(p-1)(n p-2 k)=2 k p^{2}-n p^{2}(p-1)+$ $n p^{2}(p-1)-2 k p(p-1)$.

Claim 2. Conversely, if $\Gamma_{k p+1}$ is not $p$-periodic, then $k=0 \bmod (p), k=$ $-1 \bmod (p)$, or the interval $[(2 k+3) / p,(2 k+2) /(p-1)]$ contains an integer.

Let $\Gamma_{k p+1} \supset Z / p \times Z / p$, i.e., there exists a $Z / p \times Z / p$ action on $S_{k p+1}$.

Case 1 . The $Z / p \times Z / p$ acts freely on $S_{k p+1}$. Then the Riemann-Hurwitz formula $2(k p+1)-2=p^{2}(2 h-2)$ implies $k=p(h-1)$, i.e., $k=0 \bmod (p)$.

Case 2. The $Z / p \times Z / p$ acts on $S_{k p+1}$ with two singular points. Then the Riemann-Hurwitz formula $2(k p+1)-2=p^{2}(2 h-2)+p^{2}(1-1 / p) 2$ implies $k=p(h-1)+p-1$, i.e., $k=-1 \bmod (p)$.

Case 3. The $Z / p \times Z / p$ acts on $S_{k p+1}$ with more than three singular points. Then the Riemann-Hurwitz formula $2(k p+1)-2=p^{2}(2 h-2)+p^{2}(1-1 / p) t$ implies $(2 k+t) p=(2 h-2+t) p^{2}$. Suppose $n=2 h-2+t$ and $n p=$ $(2 h-2+t) p=2 k+t \geq 2 k+3$ because of $t \geq 3$, i.e., $n \geq(2 k+3) / p$. Also, $2 k p-(2 h-2) p^{2}=p(p-1) t$, or $t=(2 k-2 h p+2 p) /(p-1)$ implies

$$
\begin{aligned}
n & =2 h-2+t=[(2 h-2)(p-1)+2 k-2 h p+2 p] /(p-1) \\
& =(2 k+2-2 h) /(p-1) \leq(2 k+2) /(p-1)
\end{aligned}
$$

since $h \geq 0$. So $(2 k+3) / p \leq n \leq(2 k+2) /(p-1)$ where $n$ is an integer.

Claim 3. $\Gamma_{k p+1}$ is not $p$-periodic if $k \geq\left(p^{2}-3\right) / 2$. Since $p \geq 3$, it follows that

$$
\begin{aligned}
(2 k+2) /(p-1)-(2 k+3) / p & =(2 k p+2 p-2 k p+2 k-3 p+3) /[p(p-1)] \\
& \geq\left(p^{2}-p\right) /[p(p-1)]=1
\end{aligned}
$$

implies that there exists at least an integer $n \in[(2 k+3) / p,(2 k+2) /(p-1)]$.

We have proved Theorem 1. 


\section{THE METACYCLIC SUBGROUPS OF THE MAPPING CLASS GROUP}

Let $M_{p, k}=\left\langle a, b \mid a^{k(p-1)}=1, b^{p}=1, a b a^{-1}=b^{s}\right\rangle$ and $N_{p, d}=\langle a, b| a^{d}=$ $\left.1, b^{p}=1, a b a^{-1}=b^{t}\right\rangle$ denote metacyclic groups, where $p$ is an odd prime, $k$ is a positive integer, $d \geq 3$ and divides $p-1, s$ and $t$ are $\bmod (p)$ integers such that the order of $s$ in the multiple group $(Z / p Z)^{*}$ is equal to $p-1$, and the order of $t$ in the multiple group $(Z / p)^{*}$ is equal to $d$. Then the order $\left|M_{p, k}\right|=k p(p-1)$ and $\left|N_{p, d}\right|=p d$.

Lemma 2.1. (a) $\Gamma_{(p-1)(k p-2-k) / 2} \supset M_{p, k}$ except for $k=1, p=3$.

(b) $\Gamma_{(p-1)(d-2) / 2} \supset N_{p, d}$.

Proof. We apply Lemma 1.1 to show $\operatorname{Homeo}^{+}\left(S_{(p-1)(k p-2-k) / 2}\right) \supset M_{p, k}$ and Homeo $^{+}\left(S_{(p-1)(d-2) / 2}\right) \supset N_{p, d}$. Write the groups $M_{p, k}=\left\langle b^{-1}, a, a^{-1} b\right\rangle \quad(h=$ $0, b=3)$ and $N_{p, d}=\left\langle b^{-1}, a, a^{-1} b\right\rangle \quad(h=0, b=3)$. Note the order $O\left(a^{-1} b\right)=k(p-1)$ in $M_{p, k}$ and the order $O\left(a^{-1} b\right)=d$ in $N_{p, d}$. It is direct to check that the Riemann-Hurwitz formula holds for both groups $M_{p, k}$ and $N_{p, d}$ :

$$
\begin{aligned}
2(p-1)(k p-2-k) / 2-2= & k p(p-1)(2(0)-2)+k p(p-1)(1-1 / p) 1 \\
& +k p(p-1)(1-1 /[k(p-1)]) / 2, \\
2(p-1)(d-1) / 2-2= & p d(2(0)-2)+p d(1-1 / p)+p d(1-1 / d) 2 .
\end{aligned}
$$

Lemma 2.2. The finite group $M_{p, k}$ (resp. $N_{p, d}$ ) is p-periodic with the p-period $2(p-1)($ resp. $2 d)$ for $k \neq 0 \bmod (p)$.

Proof. The order $\left|M_{p, k}\right|=k p(p-1)$ (resp. $\left|N_{p, d}\right|=p d$ ) implies that the group $M_{p, k}\left(\right.$ resp. $\left.N_{p, d}\right)$ does not contain $Z / p \times Z / p$ except $k=0 \bmod (p)$, i.e., $M_{p, k}$ (resp. $N_{p, d}$ ) is $p$-periodic. We will compute the $p$-period of $M_{p, k}$ (resp. $N_{p, d}$ ) by using a result of Swan [S] that states that the $p$-period of a $p$-periodic finite group equals $2\left|N\left(S_{p}\right) / C\left(S_{p}\right)\right|$ for an odd prime $p$, where $N(-)$ and $C(-)$ denote the normalizer and centralizer and $S_{p}$ is a $p$-Sylow subgroup of $G$. In fact, the order $\left|N\left(S_{p}\right) / C\left(S_{p}\right)\right|=|N(Z / p) / C(Z / p)|=$ $k p(p-1) / k p=p-1$ in $M_{p, k}$ and the order $|N(Z / p) / C(Z / p)|=p d / p=d$ in $N_{p, d}$. This completes the proof of Lemma 2.2.

The $p$-periodicity of $\Gamma_{(p-1)(k p-2-k) / 2}$ and $\Gamma_{(p-1)(d-2) / 2}$ are clear by Theorem $1(b)$. So a lower bound of the $p$-period of certain mapping class group is obtained by combining Lemmas 2.1 and 2.2.

We have thus proved Theorem 3 and the following lemma.

Lemma 2.3. The p-period of $\Gamma_{(p-1)(k p-2-k) / 2}$ is a multiple of $2(p-1)$.

\section{THE CHERN Classes OF THE CANONICAL HOMOLOGY REPRESENTATION OF THE MAPPING CLASS GROUP}

Recall that for a complex representation $f: \Gamma \rightarrow \operatorname{GL}(k, C)$ of the discrete group $\Gamma$ the Chern classes $c_{i}(f) \in H^{2 i}(\Gamma ; Z)$ are defined as Chern classes of the flat $C^{k}$-bundle over $K(\Gamma, 1)$ classified by $B f: K(\Gamma, 1) \rightarrow \operatorname{BGL}(k, C)$. Let $Q$ be a subring in $C$. If $f: \Gamma \rightarrow \operatorname{GL}(k, Q)$ is a representation over $Q$, we will write $c_{i}(f)$ for the $i$ th Chern class of the associated complex representation $\Gamma \rightarrow \mathrm{GL}(k, Q) \rightarrow \mathrm{GL}(k, C)$. 
It is well known that over $Q$ the group $Z / n Z$ has a unique faithful irreducible representation $\sigma_{n}: Z / n Z \rightarrow \mathrm{GL}(\varphi(n), Q)$, where $\varphi(n)$ is the Euler function. Glover and Mislin showed a result in [GM].

Proposition 3.1 (Glover and Mislin). Let $r: Z / p^{\alpha} \rightarrow \mathrm{GL}(k, Q)$ be a $Q$ representation. Suppose that in the decomposition of $r$ into $Q$ irreducible representation $\sigma_{p} \alpha$ occurs with multiplicity $m$, where $m$ is not divisible by $p$. Then for every $j>0,\left(C_{\varphi\left(p^{\alpha}\right)}(r)\right)^{j} \in H^{2 j \varphi\left(p^{\alpha}\right)}\left(Z / p^{\alpha} ; Z\right)$ has order $p^{\alpha}$.

Let $\mu: \Gamma_{g} \rightarrow \mathrm{Sp}(2 g, Z)$ be the map obtained by allowing a homeomorphism $h$ of $S_{g}$ to act on $H_{1}\left(S_{g} ; Z\right)$ and let $i: \operatorname{Sp}(2 g, Z) \rightarrow \operatorname{GL}(2 g, Q)$ be the canonical inclusion. Then $\varepsilon=i \mu: \Gamma_{g} \rightarrow \mathrm{GL}(2 g, Q)$ is a representation over $Q$. If $\rho: Z / p \rightarrow \Gamma_{g} \rightarrow \mathrm{GL}(2 g, Q)$ is the composite of inclusion and $\varepsilon$, and since $\rho$ is faithful, $\chi_{\rho}=m_{\rho} \chi_{\mathrm{tr}}+n_{\rho} \chi_{\sigma}$, where $\chi$ stands for the character of the representation, $\operatorname{tr}$ denotes the trivial representation, $\sigma$ is the unique irreducible representation of $Z / p$, and the integers $m_{\rho}$ and $n_{\rho}$ depend on $\rho$ [Se].

Proposition 3.2. Suppose $\Gamma_{g}$ is p-periodic for an odd prime $p, \rho=\varepsilon i: Z / p \rightarrow$ $\Gamma_{g} \rightarrow \mathrm{GL}(2 g, Q)$ is a representation of $Z / p$ over $Q$ for any inclusion $i$ : $Z / p \rightarrow \Gamma_{g}$, and $\chi_{\rho}=m_{\rho} \chi_{\mathrm{tr}}+n_{\rho} \chi_{\sigma}$. If $n_{\rho}$ is not divisible by $p$ where $i$ ranges over all inclusions, then $\Gamma_{g}$ has p-period $m_{p}$, dividing $2 \varphi(p)=2(p-1)$.

Proof. It is well known that $\Gamma_{g}$ is $p$-periodic $(p>2)$ if and only if every $p$-Sylow subgroup $S_{p}$ of $\Gamma_{g}$ is cyclic. We need to show that there exists an element $a \in \widehat{H}^{2 \varphi(p)}\left(\Gamma_{g} ; Z\right)$ such that $\operatorname{Res}(a) \in \widehat{H}^{2 \varphi(p)}(Z / p ; Z)$ is nontrivial for every $Z / p$ inclusion by the Brown-Venkov theorem.

Let $g^{*}: H^{2 \varphi(p)}\left(\Gamma_{g} ; Z\right) \rightarrow \widehat{H}^{2 \varphi(p)}\left(\Gamma_{g} ; Z\right)$ be the canonical map from the ordinary cohomology to the Farrell-Tate cohomology. The following diagram is commutative:

$$
\begin{array}{cc}
\widehat{H}^{2 \varphi(p)}\left(\Gamma_{g} ; Z\right) \stackrel{\text { Res }}{\longrightarrow} & \widehat{H}^{2 \varphi(p)}(Z / p ; Z) \\
\uparrow g^{*} & \uparrow g^{*} \\
H^{2 \varphi(p)}\left(\Gamma_{g} ; Z\right) \stackrel{\text { Res }}{\longrightarrow} H^{2 \varphi(p)}(Z / p ; Z)
\end{array}
$$

Let $a=g^{*}\left[c_{p-1}(\varepsilon)\right] \in \widehat{H}^{2 \varphi(p)}\left(\Gamma_{g} ; Z\right)$. Then $\operatorname{Res}(a)=\operatorname{Res} g^{*}\left[c_{p-1}(\varepsilon)\right]=$ $g^{*} \operatorname{Res}\left[c_{p-1}(\varepsilon)\right]=g^{*}\left[c_{p-1}(\rho)\right]$ has nontrivial order in $\widehat{H}^{2 \varphi(p)}(Z / p ; Z)=$ $H^{2 \varphi(p)}(Z / p ; Z)$ for every $Z / p$ inclusion since the irreducible representation $\sigma$ occurs with multiplicity $n$ that is not divisible by $p$, i.e., the cup-product with $g^{*}\left[c_{p-1}(\varepsilon)\right]$ gives an isomorphism for all integers $i$ and a $\Gamma_{g}$-module $Z$ : $\widehat{H}^{i}\left(\Gamma_{g} ; Z\right)_{(p)} \rightarrow \widehat{H}^{2 \varphi(p)+i}\left(\Gamma_{g} ; Z\right)_{(p)}$.

Next, we give an upper bound of the $p$-period of $\Gamma_{(p-1)(k p-2-k) / 2}$ when $k<$ $(p-1) / 2$. Consider any inclusion $i: Z / p \rightarrow \Gamma_{(p-1)(k p-2-k) / 2}$. The RiemannHurwitz formula $2(p-1)(k p-2-k) / 2-2=p(2 h-2)+(p-1) t$ implies $t=k p-k-2 p h /(p-1)=k(p-1)-s p$, where $s=2 h /(p-1)$ must be an integer. The Lefschetz-Hopf trace formula also tells us $\chi_{\rho}(T)=2-t=2-k(p-1)+s p$, where $T$ is a generator of $Z / p$ and $\rho=\varepsilon i: Z / p \rightarrow \operatorname{GL}((p-1)(k p-2-k), Q)$.

Since $\chi_{\mathrm{tr}}(1)=1, \chi_{\mathrm{tr}}(T)=1, \chi_{\sigma}(1)=p-1$, and $\chi_{\sigma}(T)=-1$, we have $\chi_{\rho}(1)=m+n(p-1)=(p-1)(k p-k-2)$ and $\chi_{\rho}(T)=m-n=2-k(p-1)+s p$. 
Then $n p=(p-1)(k p-k-2)-2+k(p-1)-s p=(k p-k-2-s) p$ implies $n=k p-k-2-s$. Notice $t \geq 0$ implies $0 \leq s \leq k-1$. If $k<(p-1) / 2$, then $(k-1) p<k p-k-k+1-2 \leq k p-k-s-2=n<k p$. So $n$ is not divisible by $p$. By Proposition 3.2 we conclude that the $p$-period of $\Gamma_{(p-1)(k p-2-k) / 2}$ divides $2(p-1)$ if $0<k<(p-1) / 2$.

We have now proved Theorem 2 by combining the results of these two sections.

\section{AN UPPER BOUND OF THE $p$-PERIOD OF A $p$-PERIODIC MAPPING CLASS GROUP $\Gamma_{g}$}

In this section, we construct a homogeneous Chern class polynomial of the canonical homology representation $\Gamma_{g} \rightarrow \mathrm{GL}(2 g, Q)$, which depends only upon the genus $g$ and the prime $p$ so that the restrictions of the homogeneous Chern class polynomial to all $Z / p$ inclusions in $\Gamma_{g}$ are nontrivial. Therefore an upper bound of the $p$-period of a $p$-periodic $\Gamma_{g}$ is obtained.

We still suppose $g>1$ and $p$ an odd prime. Let $2 g-2=m p-i$, where $0 \leq i \leq p-1$. Define a fixed point number set $B_{g, p}$ as follows:

$$
\begin{array}{ll}
B_{g, p}=\{i, i+p, i+2 p, \ldots, i+([2 g /(p-1)]-m) p\} & \text { if } i \neq 1, \\
B_{g, p}=\{1+p, 1+2 p, \ldots, 1+([2 g /(p-1)]-m) p\} & \text { if } i=1 .
\end{array}
$$

Remark. Define $B_{g, p}=\varnothing$ if in the cases (a) $i \neq 1$ and $2 p /(p-1)<m$ and (b) $i=1$ and $2 g /(p-1)<m+1$.

Lemma 4.1. If $Z / p=\langle x\rangle$ acts on the surface $S_{g}$ and $2 g-2=m p-i$, where $0 \leq i \leq p-1$, then the number $t$ of fixed points of $x$ belongs to $B_{g, p}$. Conversely, any number $t \in B_{g, p}$ can occur as the number of fixed points of an order $p$ homeomorphism $x$ on the surface $S_{g}$.

Proof. We again use Proposition 1.1. If $Z / p=\langle x\rangle$ acts on the surface, the Riemann-Hurwitz formula $2 g-2=(2 h-2) p+t p(1-1 / p)$ implies $t=$ $2(g-h) /(p-1)-2 h-2=n-(2 g-n(p-1))+2=n p-2 g+2$. Here $g-h=n(p-1) / 2, n$ is an integer, and $n \leq[2 g /(p-1)]$ since $h \geq 0$. Therefore, $t=-2 g+2=i \bmod (p)$ and $0 \leq t \leq([2 g /(p-1)]-m) p+i$, i.e., $t \in B_{g, p}$. Notice, if $i=1$ then $t \neq 1$, since the number of fixed points of $Z / p$ action cannot be 1 .

Conversely, if $t \in B_{g, p}$, i.e., $t=i+k p$, where $0 \leq k \leq[2 g /(p-1)]$ $-m$ if $i \neq 1 ; 0<k \leq[2 g /(p-1)]-m$ if $i=1$. Let $n=k+m \leq[2 g /(p-1)]$. Then $h=g-n(p-1)=g-(k+m)(p-1) / 2 \geq 0$. We show that there exists a $Z / p=\langle x\rangle$ action on the surface $S_{g}$ with $t$ fixed points numbered by Proposition 1.1. In fact, write

$$
Z / p=\langle x\rangle=\left\langle x_{1} \cdots x_{h} ; x_{1}^{-1} \cdots x_{h}^{-1} ; x_{h+1}, \ldots, x_{h+t-1}, x^{-(t-1)}\right\rangle
$$

for $t \neq 1 \bmod (p)$;

$$
Z / p=\langle x\rangle=\left\langle x_{1} \cdots x_{h} ; x_{1}^{-1} \cdots x_{h}^{-1} ; x_{h+1}^{2}, \ldots, x_{h+t-1}, x^{-t}\right\rangle
$$

for $t=1 \bmod (p)$, where $x_{j}=x, 0<j<h+t$; it is easy to check the Riemann-Hurwitz formula $(2 h-2) p+(p-1) t=(2 g-n(p-1)-2) p+$ $(p-1)(i+k p)=m p-i=2 g-2$ since $n=k+m$. 
Lemma 4.2. Let $Z / p=\langle x\rangle$ act on the surface $S_{g}$ and $\rho=\varepsilon i: Z_{p} \rightarrow \Gamma_{g} \rightarrow$ $\mathrm{GL}(2 g, Q)$ be a representation for an inclusion $i: Z_{p} \rightarrow \Gamma_{g}$. Then $\rho$ is equivalent to one of the following representations as a complex representation: $\rho_{k}=(m+k) \sigma_{p} \oplus n T r$, where $2 g-2=m p-i, 0 \leq i \leq p-1$. If $i \neq 1,0 \leq k \leq$ $[2 g /(p-1)]-m$, if $i=1,1 \leq k \leq[2 g /(p-1)]-m, n=2 g-(m+k)(p-1)$. Conversely, any representation of $\rho_{k}=(m+k) \sigma_{p} \oplus n T r$ as above can be equivalent to $\rho=\varepsilon i$ for some inclusion $i: Z / p \rightarrow \Gamma_{g}$.

Proof. It is clear that the character $\chi_{\rho_{k}}(\mathrm{Id})=(m+k)(p-1)+n=2 g$ and $\chi_{\rho_{k}}(x)=(m+k)+n=2 g-(m+k) p=2-i-k p$. On the other hand, by using the Lefschetz fixed point theorem, for any $\rho=\varepsilon i, \chi_{\rho}($ Id $)=2 g$, $\chi_{\rho}(x)=2-t \in 2-B_{g, p}=\{2-i-k p\}$. Lemma 4.1 implies $\chi_{\rho}=\chi_{\rho_{k}}$ for some $k$. Conversely, by Lemma 4.1 , there exists a $Z / p$ representation $\rho$ such that $\chi_{\rho}=\chi_{\rho_{k}}$ for every $k$. This completes the proof of Lemma 4.2.

Lemma 4.3. Let $2 g-2=m p-i$, where $p$ is an odd prime and $0 \leq i \leq p-1$. If $m<p^{r}$ then $[2 g /(p-1)]<p^{r+1}-p^{r}$.

Proof. We have $2 g-2+i=m p<p^{r+1}$, i.e., $2 g /(p-1)<\left(p^{r+1}+2\right) /(p-1)$. Since $3 p^{r+1}+2 \leq p^{r+2}+p^{r}$ implies $p^{r+1}+2 \leq p^{r+2}-2 p^{r+1}+p^{r}$, we obtain $\left(p^{r+1}+2\right) /(p-1) \leq p^{r+1}-p^{r}$.

Lemma 4.4. If $1 \leq k \leq p-1$ and $k p^{r} \leq n<(k+1) p^{r}$, then $n ! / p^{r} !\left(n-p^{r}\right) !=k$ $\bmod (p)$.

Proof. Write the integer $n ! / p^{r} !\left(n-p^{r}\right) !=\left[\left(p^{r}+1\right) /(1)\right]\left[\left(p^{r}+2\right) /(2)\right]$ $\times\left[\left(p^{r}+3\right) /(3)\right] \cdots\left[\left(p^{r}+p\right) /(p)\right]\left[\left(p^{r}+p+1\right) /(p+1)\right] \cdots\left[\left(p^{r}+p^{2}\right) /\left(p^{2}\right)\right] \cdots$ $\times\left[\left(p^{r}+p^{r}\right) /\left(p^{r}\right)\right]\left[\left(p^{r}+p^{r}+1\right) /\left(p^{r}+1\right)\right] \cdots\left[\left(p^{r}+2 p^{r}\right) /\left(2 p^{r}\right)\right]\left[\left(p^{r}+2 p^{r}+1\right) /\right.$ $\left.\left(2 p^{r}+1\right)\right] \cdots\left[\left(p^{r}+3 p^{r}\right) /\left(3 p^{r}\right)\right] \cdots\left[\left(k p^{r}\right) /(k-1) p^{r}\right] \cdots\left[(n) /\left(n-p^{r}\right)\right]$.

If $i \neq 0 \bmod (p)$ then $\left[\left(p^{r}+i\right) /(i)\right]=1$ in the field $F_{p}$.

If $i=0 \bmod \left(p^{s-1}\right)$ and $i \neq 0 \bmod \left(p^{s}\right) \quad(s \leq r)$, then $\left[\left(p^{r}+i\right) /(i)\right]=1$ in the field $F_{p}$.

If $i=m p^{r}, m=0,1,2, \ldots, k-1$, then $\left[\left(p^{r}+i\right) /(i)\right]=[(m+1) /(m)]$ in $F_{p}$.

So $n ! / p^{r} !\left(n-p^{r}\right) !=[(1)][(1)][(2) /(1)] \cdots[(k) /(k-1)]=[(k)]$ in $F_{p}$, i.e., the integer $n ! / p^{r} !\left(n-p^{r}\right) !=k \bmod (p)$ when $k p^{r} \leq n<(k+1) p^{r}$.

Proof of Theorem 4. (a) If $[2 g /(p-1)]<p^{r}$, let $\rho_{k}: Z / p \rightarrow \Gamma_{g} \rightarrow \mathrm{GL}(2 g, Q)$ be a representation equivalent to the complex $\rho_{k}=(m+k) \sigma_{p} \oplus n T r$. The total Chern class $C$ satisfies

$$
\begin{aligned}
C\left(\rho_{k}\right) & =C\left(\sigma_{p}\right)^{m+k}=\left[1+c_{p-1}\left(\sigma_{p}\right)\right]^{m+k} \\
& =\sum_{0 \leq t \leq m+k}(m+k) ! / t !(m+k-t) !\left[c_{p-1}\left(\sigma_{p}\right)\right]^{t} .
\end{aligned}
$$

So

$$
i^{*} c_{\varphi\left(p^{r}\right)}(\varepsilon)=c_{\varphi\left(p^{r}\right)}\left(\rho_{k}\right)=(m+k) ! / p^{r-1} !\left(m+k-p^{r-1}\right) !\left[c_{p-1}\left(\sigma_{p}\right)\right]^{p^{r-1}} \neq 0 \quad \bmod (p)
$$


in $H^{2 p^{r-1}(p-1)}(Z / p ; Z)$ for every inclusion $i: Z / p \rightarrow \Gamma_{g}$ since $p^{r-1} \leq m+k \leq$ $[2 g /(p-1)]<p^{r}$ by Lemma 4.4. Note that $\Gamma_{g}$ is $p$-periodic by assumption. Using an argument similar to the proof of Proposition 3.2, we obtain an upper bound $2 p^{r-1}(p-1)$ of the $p$-period of $\Gamma_{g}$ in this case.

(b) If $[2 g /(p-1)] \geq p^{r}$, consider the restriction of a homogeneous Chern class polynomial to every $Z / p$ inclusion. We have

$$
\begin{aligned}
i^{*}\{[ & \left.\left.c_{\varphi\left(p^{r}\right)}(\varepsilon)\right]^{p(p-1)}\left[c_{\varphi\left(p^{r+1}\right)}(\varepsilon)\right]^{p-1}\right\} \\
= & {\left[c_{\varphi\left(p^{r}\right)}\left(\rho_{k}\right)\right]^{p(p-1)}+\left[c_{\varphi\left(p^{r+1}\right)}\left(\rho_{k}\right)\right]^{p-1} } \\
= & {\left[(m+k) ! / p^{r-1} !\left(m+k-p^{r-1}\right) !\right]^{p(p-1)}\left[c_{p-1}\left(\sigma_{p}\right)\right]^{p^{r}(p-1)} } \\
& +\left[(m+k) ! p^{r} !\left(m+k-p^{r}\right) !\right]^{p-1}\left[c_{p-1}\left(\sigma_{p}\right)\right]^{p^{r}(p-1)}
\end{aligned}
$$

in $H^{2 p^{r}(p-1)^{2}}(Z / p ; Z)$.

If $m+k<p^{r}$, the second term above vanishes and the first term is nontrivial by Lemma 4.4 since $p^{r-1} \leq m+k<p^{r}$. If $m+k \geq p^{r}$, then $m+k \leq$ $[2 g /(p-1)]<p^{r+1}-p^{r}$ by Lemma 4.3. It implies that the second term above always equals $1 \bmod (p)$ and the first term above is 0 or $1 \bmod (p)$. So, the element $i^{*}\left\{\left[c_{\varphi\left(p^{r}\right)}(\varepsilon)\right]^{p(p-1)}+\left[c_{\varphi\left(p^{r+1}\right)}(\varepsilon)\right]^{p-1}\right\}=\left[c_{\varphi\left(p^{r}\right)}\left(\rho_{k}\right)\right]^{p(p-1)}+\left[c_{\varphi\left(p^{r+1}\right)}\left(\rho_{k}\right)\right]^{p-1}$ has order $p$ in $H^{2 p^{r}(p-1)^{2}}(Z / p ; Z)$ for every $Z / p$ inclusion. It follows that $2 p^{r}(p-1)^{2}$ is an upper bound of the $p$-period of a periodic $\Gamma_{g}$, and we have completed the proof of Theorem 4.

Remark. The upper bound of the $p$-period of $\Gamma_{g}$ in Theorem 4 is a little bit rough. It can be improved by individually computing the Chern classes of the homology representation of $\Gamma_{g}$ with the same method.

Example. Consider the 3-periodic group $\Gamma_{3}, i: Z / 3 \rightarrow \Gamma_{3}$ is an inclusion, the number of possible fixed points are 2 or 5 ; i.e., the associated representations are $\rho_{1}=2 \sigma_{3} \oplus 2 T r$ or $\rho_{2}=2 \sigma_{3}$. But $i_{1} * c_{2}(\varepsilon)=c_{2}\left(\rho_{1}\right)=c_{2}\left(2 \sigma_{3} \oplus 2 T r\right)=2 c_{2}\left(\sigma_{3}\right)$, $i * c_{6}(\varepsilon)=c_{6}\left(\rho_{1}\right)=c_{6}\left(2 \sigma_{3} \oplus 2 T r\right)=0$. Therefore, $i_{1} *\left\{\left[c_{2}(\varepsilon)\right]^{3}+c_{6}(\varepsilon)\right\}=$ $2\left[c_{2}\left(\sigma_{3}\right)\right]^{3}$ is nontrivial. Similarly, $i_{2} * c_{2}(\varepsilon)=c_{2}\left(\rho_{2}\right)=c_{2}\left(3 \sigma_{3}\right)=3 c_{2}\left(\sigma_{3}\right)=0$ $\bmod (3), i_{2} * c_{6}(\varepsilon)=c_{6}\left(\rho_{2}\right)=c_{6}\left(3 \sigma_{3}\right)=\left[c_{2}\left(\sigma_{3}\right)\right]^{3}$. So, $i_{2} *\left\{\left[c_{2}(\varepsilon)\right]^{3}+c_{6}(\varepsilon)\right\}=$ $c_{2}\left(\sigma_{3}\right)$ is nontrivial, i.e., the element $\left[c_{2}(\varepsilon)\right]^{3}+c_{6}(\varepsilon)$ is nontrivial when restricted to every $Z / 3$ subgroup. Thus, we obtain an upper bound 12 of the 3-period of $\Gamma_{3}$, which is better than the upper bound 24 given by Theorem 4 .

\section{ACKNOWLEDGMENT}

This paper is a part of the author's Ph.D. thesis at The Ohio State University. The author wishes to express the greatest thanks to his advisors Professor H. Glover and Professor G. Mislin for their excellent guidance and inspiration.

\section{REFERENCES}

[B1] K. S. Brown, Cohomology of groups, Graduate Texts in Math., vol. 87, Springer-Verlag, New York and Berlin, 1982.

[B2] _ Groups of virtually finite dimension, Proc. of Symp. 1977, Durham Conference on Homological and Combinatorial Techniques in Group Theory, pp. 27-70.

[GM] H. Glover and G. Mislin, Torsion in the mapping class group and its cohomology, J. Pure Appl. Algebra 44 (1987), 177-189. 
[H] J. Harer, The virtual cohomological dimension of the mapping class group of an orientable surface, Invent. Math. 84 (1986), 157-176.

[K] S. Kerckhoff, The Nielsen realization problem, Ann. of Math. (2) 117 (1983), 235-263.

[Se] J. P. Serre, Linear representations of finite groups, Graduate Texts in Math., vol. 42, Springer-Verlag, New York and Berlin, 1973.

[Sw] R. G. Swan, The p-period of a finite group, Illinois J. Math. 4 (1960), 341-346.

[T] T. Tucker, Finite groups acting on surfaces and the genus of a group, J. Combin. Theory Ser. B 34 (1983), 82-98.

[X] Y. Xia, Farrell-Tate cohomology of the mapping class group, Ph.D. Thesis, The Ohio State University, 1990.

Department of Mathematics, The Ohio State University, Columbus, Ohio 43210-1174 E-mail address: xia@function.mps.ohio-state.edu 